\title{
Radio Properties of AGN
}

\section{Hayk V. Abrahamyan and Areg M. Mickaelian}

\author{
Byurakan Astrophysical Observatory (BAO), Armenia \\ Email: abrahamyanhayk@gmail.com, aregmick@yahoo.com
}

\begin{abstract}
To study the radio properties of AGN, we cross-correlate and investigate Veron-Cetty \& Veron catalog of QSOs and Active Galaxies (v.13, 2010) with a number of radio catalogs: NVSS, FIRST, GB6, 87GB, SUMSS, WISH, WENSS, and 7C. This catalog contains 168,940 objects with positional accuracy of mostly 1 arcsec, though many positions have larger errors. We use new cross-correlation software based on accuracy of each object independently. In this software we take into account errors for each source and take identifications with errors within 3 sigma. Altogether, we find $\sim 16,000$ AGN having radio detection in any of the listed catalogs. Using all data from radio catalogs, we derive a homogeneous sample of radio AGN. The sample allows accomplish several tasks, including study of the distribution of radio sources by activity types, differences in physical properties of radio-loud and radio-quiet AGN, luminosity functions for various types of radio AGN, study of the q parameter by AGN types and its evolution, etc.
\end{abstract}

Keywords. radio catalogs, cross-correlations, NVSS, FIRST, QSO, blazars, AGN

To study radio galaxies and radio-loud QSOs, one needs a homogeneous sample of such objects. Most of the astrophysical problems related to AGN also include their radio emission, from the fraction of radio loud AGN among all (to match the unified scheme) to radio properties of various types of active galaxies. The main source for active galaxies is the Catalogue of QSOs and Active Galaxies (version 13, Veron-Cetty \& Veron 2010; hereafter VCV-13). It contains 168,940 objects, including 133,336 QSOs, 1,374 BL Lacs, 34,320 other AGN.

We have cross-correlated VCV-13 with GB6 (Gregory et al. 1996), 87GB (Gregory \& Condon 1991), NVSS (Condon et al. 1998), FIRST (Becker et al. 2012), SUMSS (Mauch et al. 2003), WISH (De Breuck et al. 2002), WENSS (de Bruyn et al. 1998), and 7C (Hales et al. 2007) radio catalogs. We have used a new cross-correlation software (http://arvo.sci.am/crosscorrelation/crosscor.html) allowing matching objects by their individual positional errors to avoid misidentifications and missing genuine identifications. Only identifications having positional differences less than $3 \sigma$ of errors for the given source were taken. The listed ones are the largest radio catalogs, being either all-sky or large-area ones, spanning radio frequencies from $5 \mathrm{GHz}$ to $151 \mathrm{MHz}$ (wavelengths from 6 to $198 \mathrm{~cm}$ ) and containing dozens of thousands radio sources particulalry at high galactic latitudes. NVSS contains the largest number of sources $(1,773,484)$ and FIRST contains 946,464 sources and is the deepest radio catalog having highest density of sources per $\operatorname{deg}^{2}(95)$. Both give fluxes at $1.4 \mathrm{GHz}(21 \mathrm{~cm})$. These two catalogs gave an opportunity to have large number of radio loud AGN: 15,940 identification in NVSS and 13,567 ones in FIRST. The other radio catalogs have significantly smaller number of identifications in VCV-13. In Fig. 1 the distribution of sources vs. identification radius in NVSS and FIRST is shown. The NVSS sample sources typically have identification radius less than 6 arcseconds and the FIRST sample sources have a typical identification radius less than 2 arcsecond.

Having information from GB6, 87GB, NVSS, FIRST, SUMSS, WISH, WENSS, and $7 \mathrm{C}$ catalogs, we have built the radio spectrum (radio spectral energy distribution, SED). 

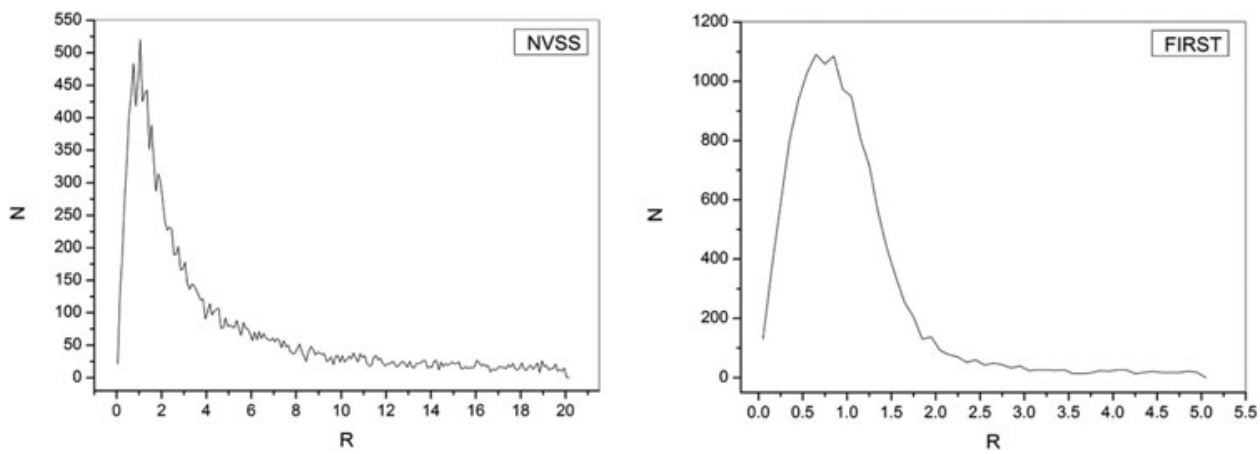

Figure 1. Distribution of identified sources by identification radius for NVSS and FIRST.

The analysis of the radio SEDs shows that there is a dip at the frequency $102 \mathrm{MHz}$ for almost all sources, which most probably could be explained by the effect of synchrotron self-absorption. For some radio galaxies, the radio spectrum is divided into three parts. These radio sources are very rare, and in the future we plan a detailed study of these objects. All spectra have been evaluated using the spectral indices of these objects, as well as preliminary grouping of radio SEDs have been accomplished.

The sample of radio loud AGN allows accomplishing several tasks:

- Determination of AGN types and the fraction of radio-loud AGN among all: blazars, QSOs, Sy1/Sy1.2/Sy1.5, Sy1.8/Sy1.9/Sy2, LINERs, composite AGN, HII regions

- Study of differences in physical properties of radio-loud and radio-quiet AGN

- Building luminosity functions for various types of radio loud AGN and studying their evolution

- Study of the q parameter by AGN types and its evolution

- FRI and FRII sources among different types of AGN and study of the fine radio structure (when possible)

- Radio and optical variability of QSOs and other AGN

The sample will be complemented by data from other wavelengths (from X-ray to FIR) to have multiwavalength SED and match with the radio properties of AGN.

\section{References}

Ahn, C. P., Alexandroff, R., Allende Prieto, C., et al. 2013, ApJS, in press.

Becker R. H., Helfand D. J., White R. L., et al. 2012, ApJ 475, 479 (1997), Vizier online version 2012Feb16

Condon J. J., Cotton W. D., Greisen E. W., et al. 1998, AJ 115, 1693

Cutri R. M., Wright E. L., Conrow T., et al. 2012, WISE All-Sky Data Release, IPAC/Caltech, VizieR On-line Data Catalog II/311

De Breuck, C., Tang, Y., de Bruyn, A. G., et al. 2002, A\&\&A 394, 59, Vizier online catalog $\mathrm{VIII} / 69$

de Bruyn, G., Miley, G., Rengelink, R., et al. 2000, NFRA/ASTRON and Leiden Observatory, Vizier online catalog VIII/62

Gregory, P. C. \& Condon, J. J. 1991, ApJS 75, 1011

Gregory, P. C., Scott, W. K., Douglas, K., \& Condon, J. J. 1996, ApJS 103, 427

Hales, S. E. G.., Riley, J. M., Waldram, E. M., et al. 2007, MNRAS 382, 1639

Massaro E., Giommi P., Leto C., et al. 2009, A\&A 495, 691

Mauch, T., Murphy, T., Buttery, H. J., et al. 2003, MNRAS 342, 1117

McMahon, R. G., Irwin, M. J., \& Maddox, S. J., 2000, IoA, Cambridge, UK

Veron-Cetty M. P. \& Veron P. 2010, A\&SA 518, A10 